\title{
Update on Exercise and Weight Control
}

\author{
Éric Doucet, ${ }^{1}$ Neil King, ${ }^{2}$ James A. Levine, ${ }^{3}$ and Robert Ross ${ }^{4}$ \\ ${ }^{1}$ Behavioural and Metabolic Research Unit (BMRU), School of Human Kinetics, University of Ottawa, \\ Ottawa, ON, Canada K1N 6N5 \\ ${ }^{2}$ Institute of Health and Biomedical Innovation, Queensland University of Technology, Brisbane, QLD, Australia \\ ${ }^{3}$ Endocrine Research Unit, Mayo Clinic, Rochester, MN, USA \\ ${ }^{4}$ School of Kinesiology and Health Studies and Division of Endocrinology and Metabolism, Queen's University, \\ Kingston, ON, Canada
}

Correspondence should be addressed to Éric Doucet, edoucet@uottawa.ca

Received 27 October 2011; Accepted 27 October 2011

Copyright (๑) 2011 Éric Doucet et al. This is an open access article distributed under the Creative Commons Attribution License, which permits unrestricted use, distribution, and reproduction in any medium, provided the original work is properly cited.

Recent analyses of population data reveal that obesity rates continue to rise and are projected to reach unprecedented levels over the next decade [1]. Despite concerted efforts to impede obesity progression, as of today, weight loss and weight maintenance strategies remain at best partially successful endeavours. Regardless of the observation that weight loss strategies can produce significant weight loss [2] and substantial improvements of the determinants of the metabolic risk profile $[3,4]$, it is clear that actual weight loss tends to be lower than the anticipated weight loss, and most individuals who achieve weight loss will likely regain some weight [5] and even overshoot [6] their preintervention body weight. As such, an improved understanding of the factors that contribute to lower than expected weight loss and poor weight maintenance would improve the effectiveness of weight loss interventions.

Increasing physical activity participation is frequently recommended as a method of improving weight management for its recognized ability to positively impact metabolic [7] and psychological health [8-10]. Despite these valuable outcomes, weight loss is often much less than expected, when exercise is employed as the sole means of intervention [11] at least as far as effectiveness is concerned. This suggests that, amongst other things, compensatory responses (i.e., increased energy intake and/or reduced nonstructured physical activity) occur which undermine the weight loss. It is possible that these compensatory responses are driven at least in part by the observations that weight loss and exercise increase appetite and the reinforcing value of foods [12, 13] and that exercise has also been shown to decrease nonexercise energy expenditure [14]. Exercise can also lead to beneficial changes in ectopic fat storage even in the absence of major changes in body weight [15], an outcome that may be easily overlooked with traditional markers of weight loss success.

In this special issue we sought papers related to exerciseinduced weight loss with a particular emphasis on the effects of exercise on ectopic fat mobilization, non-exercise activity thermogenesis, and appetite regulation. This special issue contains 14 original manuscripts and 5 review articles that cover a wide array of methodologies, populations, training modalities but that all share the common feature of dealing with different aspects of physical activity/exercise and weight management.

Five comprehensive reviews appear in this special issue. In the paper by B. Strasser and W. Schobersberger "Evidence for resistance training as a treatment therapy in obesity", clear recommendations on the use of resistance training for the treatment of obesity were derived from available literature. J. Nantel et al. present in "Physical activity and obesity: biomechanical and physiological key concepts" an interesting overview of the biomechanical considerations related to physical activity and obesity whereas the paper by S. H. Boutcher "High-intensity intermittent exercise and fat loss" provides arguments for increasing exercise intensity to improve the effects of exercise on fat mobilization and metabolic health. The fourth review article by J. P. Chaput et al. "Physical activity plays an important role in body weight regulation" presents a series of studies that provide evidence 
in support of an important role of physical activity in body weight control. In the last of the reviews "Cognitivebehavioral strategies to increase the adherence to exercise in the management of obesity", R. D. Grave et al. surveyed the literature on cognitive-behavioural strategies to increase the adherence to exercise in hopes of improving weight management.

The special issue included three original research communications that dealt directly with eating behaviour and appetite. Results presented in "Low fat loss response after medium-term supervised exercise in obese is associated with exercise-induced increase in food reward" by G. Finlayson et al. highlight the role of hedonic processes and, in particular, increased food reward as a contributor to reduced fat loss in response to exercise. The paper "Influence of physical activity participation on the associations between eating behaviour traits and body mass index in healthy postmenopausal women" by M. E. Riou et al. presents evidence that the relationship between eating behaviour traits and adiposity may well be modulated by the level of physical activity. In the third paper "The acute effects of swimming on appetite, food intake, and plasma acylated ghrelin," J. A. King et al. report that moderate intensity swimming increases appetite, but that this effect is not related to circulating ghrelin levels.

Two additional research communications are directly in line with this special issue. The first one "Acute impact of moderate-intensity and vigorous-intensity exercise bouts on daily physical activity energy expenditure in postmenopausal women" presents results that support the notion that total physical activity energy expenditure may actually be lower on days where structured exercise is performed in obese women, an effect that seems to be accentuated for high intensity exercise. Results in "Impact of weight loss on physical function with changes in strength, muscle mass, and muscle fat infiltration in overweight to moderately obese older adults: a randomized clinical trial" from an exercise intervention with or without a weight loss component showed that muscle fat infiltration decreased significantly in both groups but to a much greater extent in the exercisers that also experienced weight loss.

This special issue also contains 4 original research communications that relate the effects of exercise and weight control on psychological outcomes. In the paper "Predictors of psychological well-being during behavioral obesity treatment in women" by P. N. Vieira et al., the results support that self-determined motivation for exercise seems to positively impact some psychological variables, an effect that is independent of weight change during the weight loss intervention. Amongst other things, the paper "Behavioral and psychological factors associated with 12-month weight change in a physical activity trial" by M. A. Napolitano and S. Hayes highlighted that the success of long-term weight loss was related to improved self-efficacy whereas weight gain was associated to depressed mood. Results by A. J. Green et al. paper "Impact of regular exercise and attempted weight loss on quality of life among adults with and without type 2 diabetes mellitus" published in this issue show that exercise does not exert the same effects on quality of life in individuals with type 2 diabetes. The last paper in this group "Effectiveness of a home-based postal and telephone physical activity and nutrition pilot program for seniors" investigated the effects of a 12-week home-based physical activity and nutrition intervention in seniors. The program was successful at increasing walking time which was partly attributable to increased awareness of health and well-being.

Three original papers related to children appear in this special issue. In one of these papers "Utility of accelerometers to measure physical activity in children attending an obesity treatment intervention" reported, W. Robertson tested the utility of accelerometers to assess physical activity in children attending an obesity treatment intervention. The results highlight the possibility that accelerometers may underestimate physical activity in this population. Results from another study reported in the paper "How children move: activity pattern characteristics in lean and obese chinese children"different physical activity patterns measured with accelerometry between lean and obese children in the paper. "A 10-month physical activity intervention improves body composition in young black boys," an after-school physical activity program was shown to improve body composition and fitness in African-American boys who attended at least 3 times per week.

The last two communications relate to levels of physical activity. In the first of these 2 papers "SALSA: sAving lives staying active to promote physical activity and healthy eating," an 8-week Latin dance intervention was shown to increase physical activity in overweight women. Results of the last study in this issue "Population-based estimates of physical activity for adults with type 2 diabetes: a cautionary tale of potential confounding by weight status" suggest that estimates of physical activity levels vary by BMI categories, which may lead to misclassify adults with type 2 diabetes as being sufficiently active.

In summary, the original research combined with the review papers in this special issue highlight the fact that research on exercise and body weight control is evolving. The breadth of research questions around this topic will, in time, help us understand the multifaceted aspects of the effects of exercise on the management of body weight and the complications associated with increased adiposity.

\section{Éric Doucet Neil King \\ James A. Levine Robert Ross}

\section{References}

[1] Y. C. Wang, K. McPherson, T. Marsh, S. L. Gortmaker, and M. Brown, "Health and economic burden of the projected obesity trends in the USA and the UK," The Lancet, vol. 378, no. 9793, pp. 815-825, 2011.

[2] T. A. Wadden, "Treatment of obesity by moderate and severe caloric restriction: results of clinical research trials," Annals of Internal Medicine, vol. 119, no. 7, part 2, pp. 688-693, 1993.

[3] A. Tremblay, E. Doucet, P. Imbeault, P. Mauriège, J. P. Després, and D. Richard, "Metabolic fitness in active reduced-obese individuals," Obesity Research, vol. 7, no. 6, pp. 556-563, 1999. 
[4] R. R. Wing and R. W. Jeffery, "Effect of modest weight loss on changes in cardiovascular risk factors: are there differences between men and women or between weight loss and maintenance?" International Journal of Obesity, vol. 19, no. 1, pp. 67-73, 1995.

[5] R. L. Weinsier, K. M. Nelson, D. D. Hensrud, B. E. Darnell, G. R. Hunter, and Y. Schutz, "Metabolic predictors of obesity," Journal of Clinical Investigation, vol. 95, no. 3, pp. 980-985, 1995.

[6] A. G. Dulloo, J. Jacquet, and L. Girardier, "Poststarvation hyperphagia and body fat overshooting in humans: a role for feedback signals from lean and fat tissues," American Journal of Clinical Nutrition, vol. 65, no. 3, pp. 717-723, 1997.

[7] D. H. Ryan, "Risks and benefits of weight loss: challenges to obesity research," European Heart Journal, vol. 7, pp. L27-L31, 2005.

[8] V. Messier, R. Rabasa-Lhoret, E. Doucet et al., "Effects of the addition of a resistance training programme to a caloric restriction weight loss intervention on psychosocial factors in overweight and obese post-menopausal women: a Montreal Ottawa new emerging team study," Journal of Sports Sciences, vol. 28, no. 1, pp. 83-92, 2010.

[9] N. A. King, M. Hopkins, P. Caudwell, R. J. Stubbs, and J. E. Blundell, "Beneficial effects of exercise: shifting the focus from body weight to other markers of health," British Journal of Sports Medicine, vol. 43, no. 12, pp. 924-927, 2009.

[10] E. Ekeland, F. Heian, K. B. Hagen, J. Abbott, and L. Nordheim, "Exercise to improve self-esteem in children and young people," Cochrane Database of Systematic Reviews, no. 1, p. CD003683, 2004.

[11] W. C. Miller, D. M. Koceja, and E. J. Hamilton, "A metaanalysis of the past 25 years of weight loss research using diet, exercise or diet plus exercise intervention," International Journal of Obesity, vol. 21, no. 10, pp. 941-947, 1997.

[12] J. D. Cameron, G. S. Goldfield, M. J. Cyr, and E. Doucet, "The effects of prolonged caloric restriction leading to weightloss on food hedonics and reinforcement," Physiology and Behavior, vol. 94, no. 3, pp. 474-480, 2008.

[13] E. Doucet, P. Imbeault, S. St-Pierre et al., "Appetite after weight loss by energy restriction and a low-fat diet-exercise followup," International Journal of Obesity, vol. 24, no. 7, pp. 906914, 2000.

[14] R. C. Colley, A. P. Hills, N. A. King, and N. M. Byrne, "Exercise-induced energy expenditure: implications for exercise prescription and obesity," Patient Education and Counseling, vol. 79, no. 3, pp. 327-332, 2010.

[15] P. M. Janiszewski and P. R. Ross, "Physical activity in the treatment of obesity: beyond body weight reduction," Applied Physiology, Nutrition and Metabolism, vol. 32, no. 3, pp. 512522, 2007. 


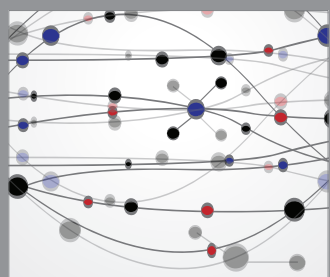

The Scientific World Journal
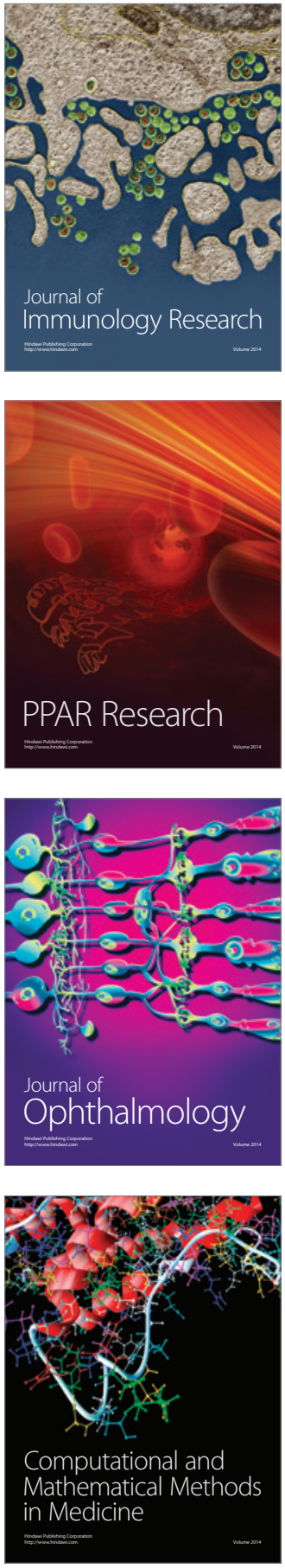

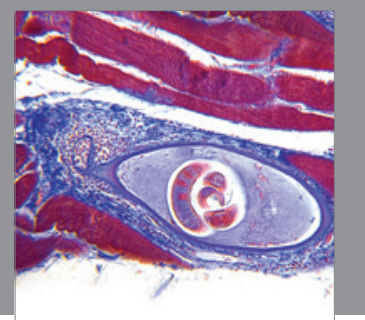

Gastroenterology

Research and Practice
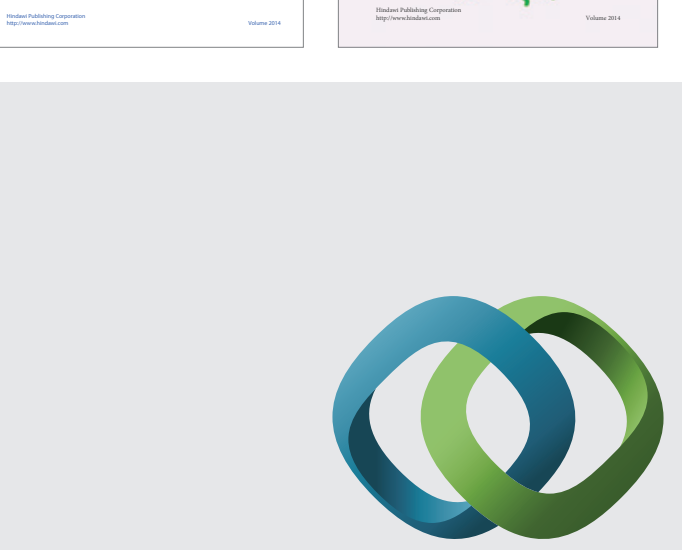

\section{Hindawi}

Submit your manuscripts at

http://www.hindawi.com
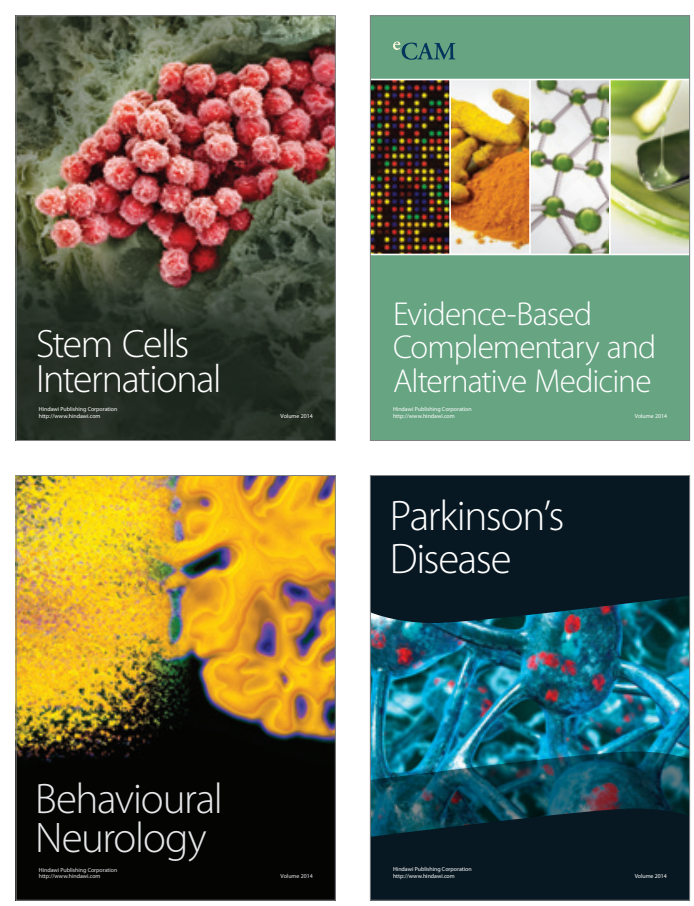

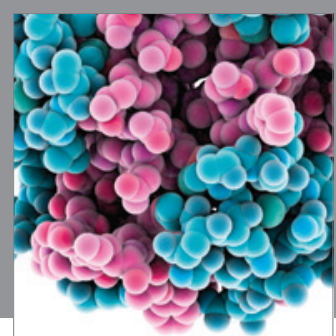

Journal of
Diabetes Research

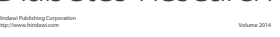

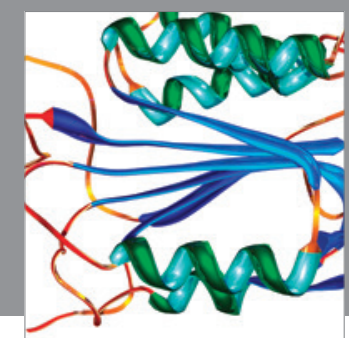

Disease Markers
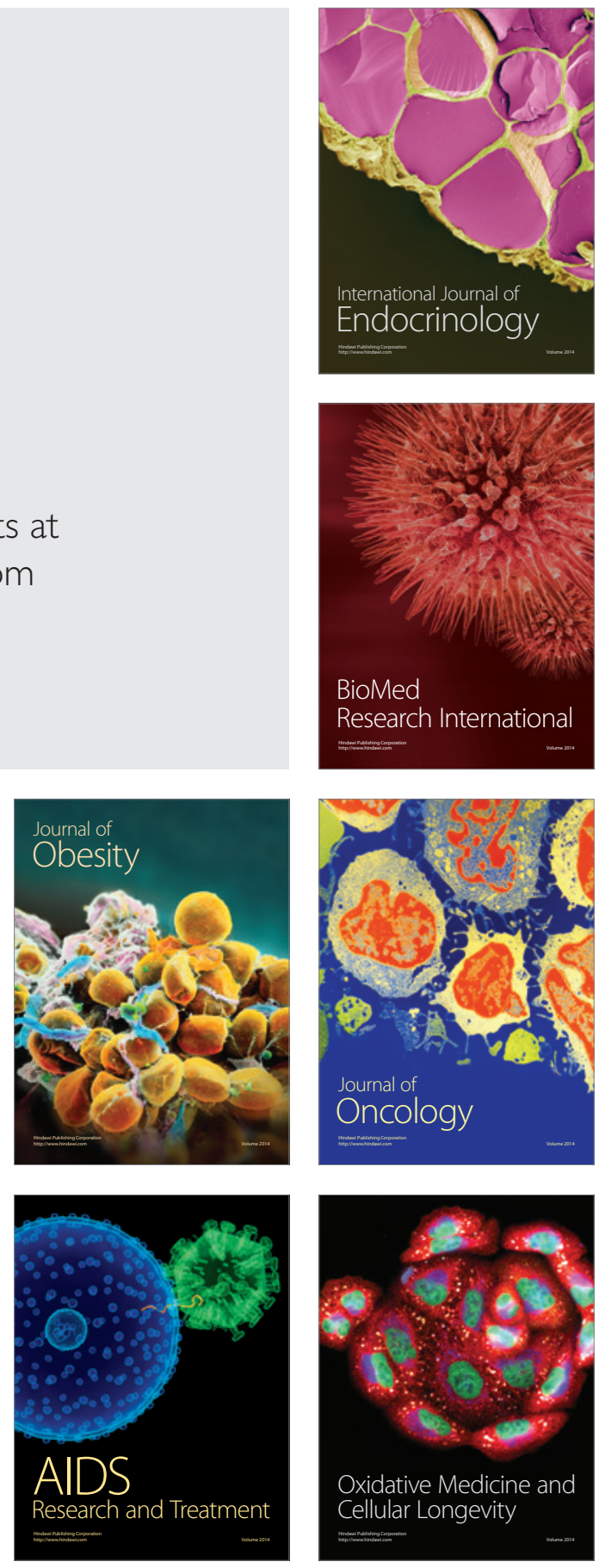